\title{
An Investigation and Comparison of Invasive Weed, Flower Pollination and Krill Evolutionary Algorithms
}

\author{
Marjan Abdeyazdan \\ Department of Computer Engineering \\ College of Electricity and Computer, \\ Mahshahr branch, \\ Islamic Azad University \\ Mahshahr, Iran
}

\author{
Samaneh Mehri Dehno \\ Department of Computer Engineering \\ College of Electricity and Computer, \\ Mahshahr branch, \\ Islamic Azad University \\ Mahshahr, Iran
}

\author{
Sayyed Hedayat Tarighinejad \\ Department of Computer Engineering \\ Yasooj Branch, \\ Islamic Azad University \\ Yasooj, Iran
}

\begin{abstract}
Being inspired by natural phenomena and available biological processes in the nature is one of the difficult methods of problem solving in computer sciences. Evolutionary methods are a set of algorithms that are inspired from the nature and are based on their evolutionary mechanisms. Unlike other optimizing methods of problem solving, evolutionary algorithms do not require any prerequisites and usually offer solutions very close to optimized answers. Based on their behavior, evolutionary algorithms are divided into two categories of biological processes based on plant behavior and animal behavior. Various evolutionary algorithms have been proposed so far to solve optimization problems, some of which include evolutionary algorithm of invasive weed and flower pollination algorithm that are inspired by plants and krill algorithm inspired by the animal algorithm of sea animals. In this paper, a comparison is made for the first time between the accuracy and rate of involvement in local optimization of these new evolutionary algorithms to identify the best algorithm in terms of efficiency. Results of various tests show that invasive weed algorithm is more efficient and accurate than flower pollination and krill algorithms.
\end{abstract}

Keywords-evolutionary algorithm; invasive weed algorithm; flower pollination algorithm; krill algorithm

\section{INTRODUCTION}

Evolution is a set of processes through which creatures have gradually learnt how to overcome the problems surrounding them and better interact with the environmental changes around them. In evolutionary processes, creatures that are more adapted to their environment are more likely to survive. Natural selection is considered one of the key terms in evolution and is defined as the process that creates different and various genes in animals over time and is known as one of the factors of formation of new species in nature. The environment surrounding these creatures can influence their characteristics and species that have become adapted to the environmental changes over time will continue living. Evolutionary algorithms are a set of algorithms inspired by laws of nature and Darwin's principles that play an important role in the form of an optimization problem in solving many practical issues of today. The complexity of real and practical issues will lead to the reduction of efficiency of traditional methods to solve such problems. The problem starts when nonlinear degree and the complexity of the atmosphere of problem solving are too difficult to be properly and efficiently solved by mathematical or gradient methods. A practical and optimization problem might be so complicated and non-linear that solving it using common methods might seem impossible to some extent. Using their dispersed population in problem solving environment, evolutionary algorithms have changed to one of the most efficient tools of such problem solving (Yang, 2010). Different evolutionary algorithms have been suggested so far for solving difficult and complicated problems. Unlike gradient-based methods, evolutionary algorithms are not deterministic and are inspired by random processes in nature. Evolutionary algorithms are a set of intelligent search algorithms that are able to search in the problem environment and be convergent with efficient answers with enough accuracy (Dasgupta and Michalewicz, 2013). Evolutionary algorithms perform based on different processes like genetics, evolution, ecosystem, swarm intelligence, etc.Charles Darwin has defined a set of fundamental laws for evolutionary rules that form the base of the science of Evolution. Evolutionary laws state that more adapted people are more likely to survive and continue their generation. Evolutionary behaviors are clear in all biological phenomena. For example, a specific kind of ringdove a kind of bird called Cuckoo) uses other birds' nests, whose eggs are similar to it, for laying eggs. In this reproduction behavior, the ringdove does not lie on its eggs and the victim bird takes care of all the eggs in its nest with the imagination that they are all its own. Ringdoves' behavior has not appeared overnight, but they have learnt over time that they can increase the probability of the survival of their generation by putting their eggs among other birds' eggs (Ghose et al., 2015). Evolutionary behaviors are clearly detectable among creatures like fireflies, dolphins, spiders, ants and bees. Evolutionary behaviors also exist among microscopic organisms like bacteria and body cells whose target is surviving and behaviors like being yokemates (Tripathy and Mishara, 2015). Plants' behaviors have also been formed reproducing. For example, bacteria have reached resistance against anti-biotic over time and this drug resistance is due to revolutionary based on evolution and natural selection. Getting flowers for reproduction or photosynthesis to produce sugar are some examples. Plants' behavior for surviving and adapting to their environment is one of the interesting evolutionary behaviors in nature. Plants compete with other plants to gain different resources such as water, soil and sunlight to survive. One of evolutionary algorithms that is formed based on plants behaviors and the competition between them is invasive weed algorithm (Mehrabian and Lucas, 2006). In this algorithm, any plant that is more adapted is more likely to survive by producing more seeds. Flower pollination algorithm is also an 
evolutionary algorithm based on plant behavior that flowers' pollination is considered a vital issue in survival of flowers and consequently reproduction of the plant (Yang, 2012). Evolution-based behaviors are not just limited to animals and plants, but a set of behaviors in physical phenomena show rules and principles to create nature-based algorithms. For example, water drop behavior on river paths can be modeled using physics rules and principles and this behavior can be used today to solve the difficult problems. Being inspired by different behaviors of creatures like social interactions and human emotions can inspire formation of evolutionary methods.

Evolutionary algorithms attempt to use biological, social and natural processes to solve difficult problems and overcome available challenges like these phenomena. A wide range of evolutionary algorithms have been proposed today that indicate the significance of this computing branch in computer sciences. In this paper, the evolutionary algorithms of invasive weed and flower pollination as algorithms inspired by plants and krill algorithm as the evolutionary algorithm of animals (Alavi and Gandomi, 2012) will be investigated and in the following the accuracy and convergence of these evolutionary algorithms will be compared using a set of benchmark functions.

\section{A. Invasive Weed Algorithm}

Invasive weeds in a common and single definition are plants that are not the aim of farmers but they are growing on the farms. Any tree, bush, shrub or plant branch or leaf might be recognized as invasive weed. Invasive weeds are highly adaptive in growth and reproduction on farms. Their growth prevents from the growth of products and waste of resources such as soil, water and fertilizers. The life style of plants and invasive weeds follows a specific cycle of reproduction. Invasive weeds produce a specific number of seeds according to the properties and suitability of the plant and then the seeds are transformed into invasive weeds that compete with each other in absorbing water, sunlight, and soil and so on and only stronger and more adaptive plants survive. The interesting and yet simple mechanism of invasive weed reproduction has led to the invention of an algorithm based on these plants that is called Invasive Weed Optimization (IWO) (Mehrabian and Lucas, 2006). Invasive weed algorithm has been modeled based on the reproduction cycle of invasive weeds. In this algorithm, first plant seeds are scattered randomly in the problem space and the primary invasive weeds are formed by the growth of the seeds and each invasive weed creates a number of seeds around it depending on its fitness and these seeds grow and reproduce to compete with mother and other plants. Modeling plant behavior, the invasive weed algorithm attempts to solve the difficult and optimization problems. The general stages of this algorithm are as follows:

- Each plant scatters a number of seeds in the environment based on its fitness.

- The level of seed production around each plant is defined according to the fitness of that plant.

- Transmittal of seeds around parent plants with normal distribution.
- Seeds growth and production of new plans and investigation of plant fitness.

- Repetition of previous stages to reach proper convergence.

Invasive weed algorithm makes use of a multi-step and repetitive process to reach the desired answers to solve different optimization problems. This algorithm defines a fitness for each plant that can be defined based on the maximum or minimum of objective function.

First, each plant scatters a number of seeds in the environment based on its fitness. The number of seeds produced is defined according to the fitness of the plant. In figure (1), a linear relationship (the simplest pattern of seed production) is used to calculate the number of produced seeds around each plant. As it is shown in the figure below, the maximum number of seeds is produced by colony and invasive weed that had the most fitness. Similarly, the minimum number of seeds is produced by the invasive weed that had the least fitness (Mehrabian and Lucas, 2006).

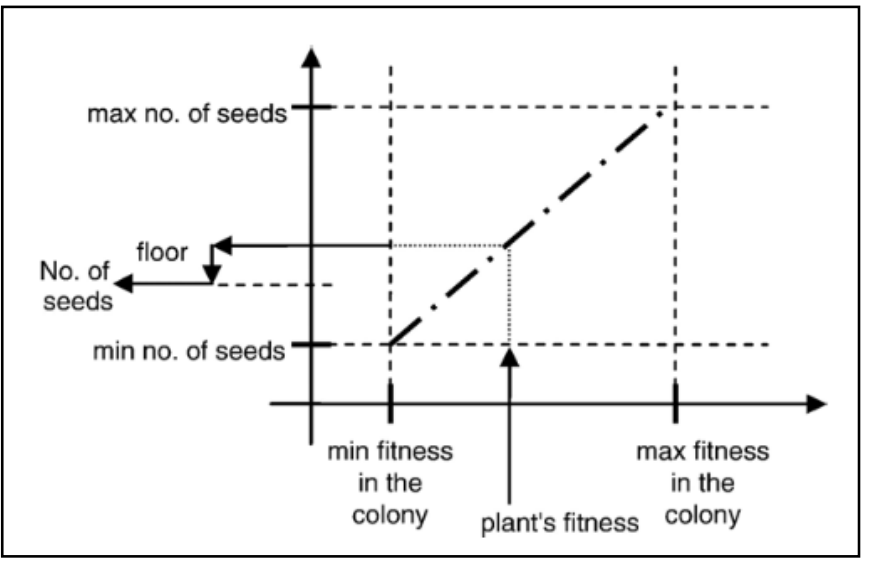

Fig. 1. Linear relationship between fitness and seed production of an invasive weed

In order to calculate the number of produced seeds by plant $\mathrm{C}$, it suffices to write the line equation of figure (1) that passes through initial and final points as it is shown in equation (1).

Equation (1)

$$
\begin{gathered}
s=s_{\min }+\frac{s_{\max }-s_{\text {min }}}{\text { fitnes }_{\max }-\text { fitnes }_{\min }} \text { (fitness } \\
\left.- \text { fitness }_{\min }\right)
\end{gathered}
$$

In this equation, smax, smin, fitnessmax and fitnessmin are maximum number of produced seeds, minimum number of produced seeds, maximum fitness of invasive weed and minimum fitness of invasive weed, respectively. $S$ is the number of seeds produced and fitness is the level of fitness of an invasive weed that target seed has produced.

Transmittal of seeds around the parent plant is one of the significant stages of invasive weed algorithm. Seed transmittal can be considered based on a normal distribution because it can properly model distribution behavior of random and natural phenomena. 


\section{B. Flower Pollination Algorithm}

There are about 250 thousand plant species and about 80 percent of them can produce flowers. It has been about 125 million years since the evolution of flowers in cretaceous period and imagining a world with plants without flowers is impossible. Flowers can be considered sexual organs of reproduction in flowering plants. Flowers play a crucial role in pollination to reproduce plants. Flower pollination is done in different methods like insects, birds or other animals. There are a number of plants that only let insects and animals do the pollination; that is, pollination in these plants takes place in a special and advanced way. About 90 percent of flowering plants need creatures like birds and insects for pollination and only 10 percent of them do not require insects or birds for pollination. Plants like willow and grass are only dependent on wind and rain for pollination. Therefore, they do not have petals to attract insects and their flower organs are simple. Pollination by insects such as honey bees is usually done only on one flower specie and they guarantee the reproduction of that flowering plant and increase the probability of its survival. Pollination of a flower by a specific insect with short memory that is able to learn only a limited amount will make the insect focus on a few number of flowers to find food and search less. On the other hand, searching for plants with good nectar for the insect might be time consuming and costly. Two types of pollination namely self-pollination and cross pollination can be observed in flowering plants. In self-pollination, pollens of a flower from a plant are placed on another flower of it and in cross pollination, pollens of one flower from one plant are placed on the flower of another plant. An example of selfpollination is in peach plant, in which the pollens of one flower are placed on another flower of the same tree. In global (cross) pollination, pollens of flowering plants are taken to different distances by insects. Considering the characteristics of pollination in flowering plants, the following four simple rules can be presented for modeling the algorithm (Yang, 2012):

1) Since cross pollination is done by insects flying pollens of flowers, it is considered as global pollination.

2) Self-pollination is considered a local pollination.

3) The probability of flower constancy is presented in a probability function of the similarity of the flower that has pollinated with this flower.

4) Selection of local pollination or global pollination of a flower is considered a probability in the $\mathrm{p} \in[0,1]$ interval.

The global pollination of a flower is modeled in a mathematical equation in equation (2):

Equation (2):

$$
x_{i}^{t+1}=x_{i}^{t}+L\left(x_{i}^{t}-g_{*}\right)
$$

In this equation, $\boldsymbol{x}_{\boldsymbol{i}}^{\boldsymbol{t}}, \boldsymbol{x}_{\boldsymbol{i}}^{\boldsymbol{t + 1}}, \boldsymbol{g}_{*}$ and $\mathrm{L}$ are the ith place of pollen in the th replication, the ith place of pollen in $t+1$ th replication, the best place of pollen found so far and pollination power that show the direction and jumping of pollens, respectively. Pollen $\mathrm{i}$ or the solution vector of xi are the best solutions so far for $\mathrm{t}$ and $\boldsymbol{g}_{*}$ among all the current solutions of the current/repetition generation. Parameter $\mathrm{L}$ is pollination power which in fact is a step in equation (3) that has shown this probability distribution.
Equation (3):

$$
\mathbf{L} \sim \frac{\lambda \Gamma(\lambda) \sin \left(\frac{\pi \lambda}{2}\right)}{\pi} \times \frac{1}{s^{1+\lambda}}, s>s_{0}>0
$$

In this equation, $\Gamma(\lambda)$ is the standard gamma function and the appropriate value for this function is $\lambda=1.5$. Local pollination or self-pollination of the flowers can also be defined using equation (4).

Equation (4):

$$
x_{i}^{t+1}=x_{i}^{t}+\varepsilon\left(x_{j}^{t}-x_{k}^{t}\right)
$$

In this equation, $x t j$ and $x t k$ are two different groups that are formed by similar flowers.

\section{Krill ALGORITHM}

FORMATION of categories and groups between sea animals is not merely a random phenomenon and many studies have been carried out on it (Gharavian et al., 2013). Living in groups or herds allows sea creatures to confuse attackers and on the other hand, leads them towards food sources. Many mathematical models have been proposed to describe the behavior of creatures that live in groups or herds (Wang et al, 2014). One of the creatures that live in great groups is Antarctic Krill that are sometimes referred to as Free Sea Krill. These creatures are capable of creating a group with 10 to 100 meters of radius in a short period of time and they can even join other groups and create even bigger groups. Each one of these groups can travel the sea or ocean in parallel. Many creatures like seals, penguins and birds attack krill. The purpose of attackers is to scatter these creatures from their group or herd and hunt them easily. Results of experimental cstudies have shown that hunting krill that are not in the herd is easy, while when the krill are moving in groups, the attackers will be confused due to krill' parallel motions and consequently the probability of hunting them decreases. When the group or herd of krill is attacked by attackers, their herd is destroyed and they tend to join the closes herd or group to decrease the probability of being hunted. In general, creating a herd or group by krill is a multi-purpose process, whose two purposes are stated bellow (Gandomi and Alavi, 2012).

- Increasing krill density

- Reaching food

Krill algorithm is a metaheuristic algorithm based on the behavior of increasing density and searching for food that is modeled to solve optimization problems. Krill algorithm tries to guide these creatures to places with higher density and more food. The objective function in krill algorithm is modeled in an area using krill density and amount of food. I krill algorithm, the global optimized points are the ones that include high density of krill and high amount of food that krill are finally guided there. Three main behaviors are considered for the motion of krill in krill algorithm (Gandomi and Alavi, 2012):

- Motion included by other krill individuals

- Foraging activity 


\section{- Random diffusion}

Each krill moving in searching spaces of the problem is stated and modeled based on a lagrangian model that is shown in equation (5)

Equation (5):

$$
\frac{d X_{i}}{d t}=N_{i}+F_{i}+D_{i}
$$

Where, Ni, Fi and Di are the motion of ith krill to other individual krill, motion of ith krill for foraging and random motion of ith krill in searching spaces of problem. In this equation, $\mathrm{Xi}$ is the location vector of ith krill and $\frac{d X_{i}}{d t}$ is the speed of the ith krill at th time. Individual krill can move according to equation (6). In fact, the effect of repulsion and attraction of each krill on other specific krill is modeled in this equation (Gandomi and Alavi, 2012).

Equation (6):

$$
N_{i}^{n e w}=N^{\max } \alpha_{i}+\omega_{n} N_{i}^{o l d}
$$

Where, $\boldsymbol{N}_{i}^{\text {new }}$ is the speed and motion of the new i krill in problem space.

$\boldsymbol{N}^{\text {max }}$ : The maximum possible speed of an individual krill and experimentally it is commonly measured to be $\boldsymbol{N}^{\max }=$ $0.01 m s^{-1}$

$\boldsymbol{N}_{i}^{\text {old }}$ : The previous speed and motion of ith individual krill in problem space

$\boldsymbol{\alpha}_{\boldsymbol{i}}$ : Direction of ith individual krill in problem space

$\boldsymbol{\omega}_{\boldsymbol{n}}$ : Inertia and weight of ith individual krill in problem space that is commonly a random number between zero and one.

$\boldsymbol{\alpha}_{\boldsymbol{i}}$ : Local effect of ith individual krill that shows the angle of krill motion for local search.

Foraging motion of krill refers to the state in which krill move towards points with more food. In fact, minimization of the distance between the krill and food is an objective function for krill in foraging food. Krill use two important factors of food location and previous experience about the food location in foraging food. Motion vector of ith krill is defined in equation (7).

Equation (7):

$$
F_{i}=V_{f} \cdot \beta_{i}+\omega_{f} \cdot F_{i}^{o l d}
$$

Where,

$\boldsymbol{V}_{\boldsymbol{f}}$ : Foraging speed and is usually considered to be $V_{f}=0.02 m s^{-1}$

$\boldsymbol{\omega}_{f}$ : Inertia weight in foraging food.

$\boldsymbol{F}_{\boldsymbol{i}}^{\text {old }}$ : Last foraging motion value

$\boldsymbol{F}_{\boldsymbol{i}}$ : Speed vector that is being used in foraging right now

Apart from motions influenced by other krill and foraging motions, each krill has another random motion that is called physical diffusion or random motion. The random motion of each krill can be shown in maximum speed value and a random vector like equation (8).

Equation (8):

$$
D_{i}=D^{\max } \delta
$$

Where,

$D^{\max }$ and $\delta$ are maximum speed and random value between $[-1,+1]$. The appropriate value for maximum speed has been determined to be $D^{\max } \in[0.002,0.01]$ (Gandomi and Alavi, 2012).

Having modeled the triple motions of the krill, the direction of krill motions can be determined in period from $\mathrm{t}$ to $\mathrm{t}+\Delta \mathrm{t}$ using equation (9).

Equation (9):

$$
X_{i}(t+\Delta t)=X_{i}(t)+\Delta t \frac{d X_{i}}{d t}
$$

\section{FINDINGS}

One of the methods of evaluating evolutionary algorithm efficiency is using mathematical evaluative functions, four of which are presented in table (1) along with the formulas and target range (Moré and Wild, 2009).

TABLE I. Evaluative Functions USED IN THE RESEARCH

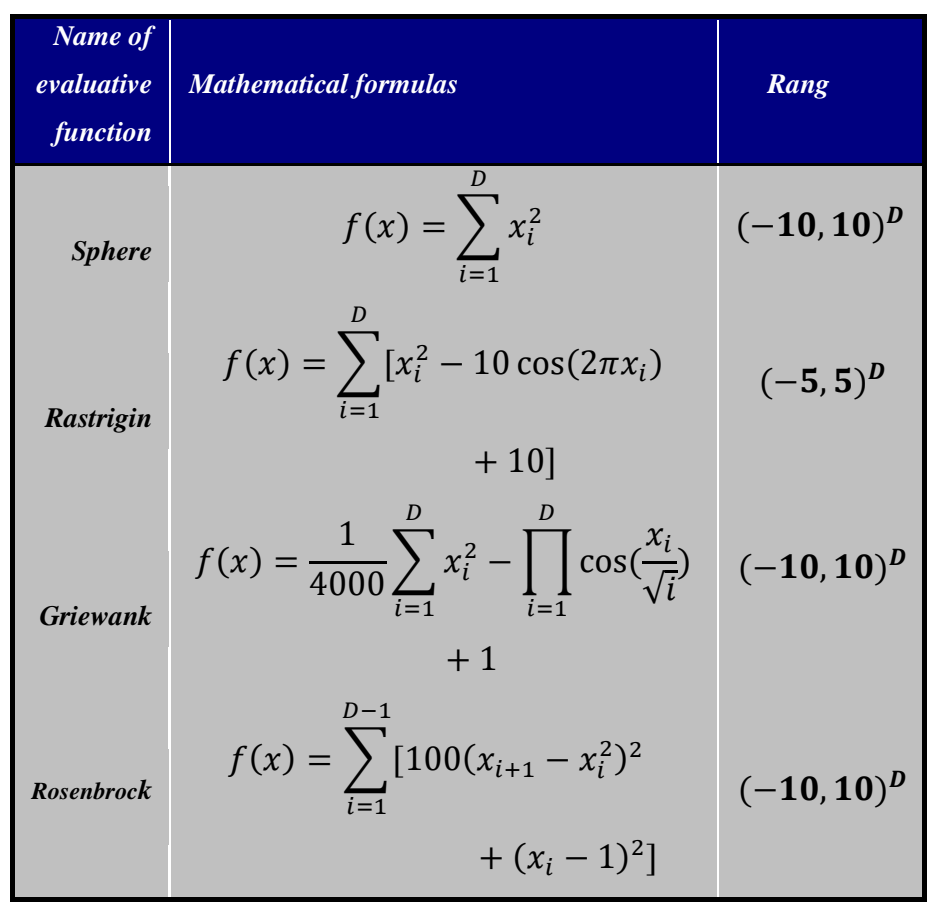

Sphere and Rosenbrock evaluative functions have global minimums and Griewank and Rastrigin evaluative functions have local minimums apart from having global minimums.

Sphere and Rosenbrock have simpler forms compared to Rastrigin and Griewank evaluative functions. Complexity of an evaluative function indicates that it is a more difficult evaluative criterion. The efficiency of evolutionary algorithms 
is higher when they are more convergent with global minimum than local minimum and also when they find the global minimum more accurately in problem space. Invasive weed, flower pollination and krill evolutionary algorithms are carefully assessed and compared in this part and results obtained from 50 different tests for the three algorithms of invasive weed, flower pollination and krill have been applied on Sphere, Rastrigin, Griewank and Rosenbrock evaluative functions with initial populations of 40 and replications of 50, 40, 30, 20 and 100, respectively. The chart in figure (2) shows the convergence of these evolutionary algorithms for each replication. As the convergence chart of the three algorithms show, the invasive weed algorithm is faster optimized to the desired answers than flower pollination and krill algorithms. On the other hand, the level of convergence of flower pollination algorithm is better than that of Antarctic krill algorithm.

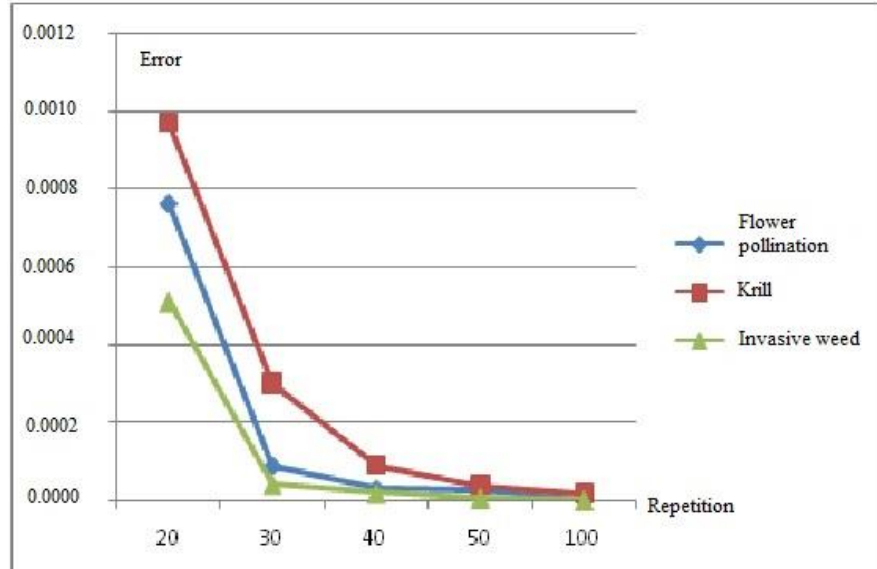

Fig. 2. Comparison of convergence of invasive weed, flower pollination and krill algorithms

Rastrigin and Griewank evaluative functions have local optimizations and there is the possibility that an evolutionary algorithm be convergent to the local optimization instead of being convergent to global optimization. The reduction in convergence rate to local optimizations is one of the most significant indices of a good and accurate evolutionary algorithm. One of the criteria of comparison between evolutionary algorithms is the tendency to convergence to global optimization answers and being away from local optimizations. For example, invasive weed algorithm can properly calculate the global optimization at $x=0.004, y=$ $0.000, f_{\min }=0.005$ in Rastrigin evaluative function. While krill algorithm has been converged to a local optimization at $x=0.9952, y=1.9898, f_{\text {min }}=4.9748$. the percentage of being involved in local optimization for Grienwank evaluative function by invasive weed, flower pollination and krill algorithms is $\% 11, \% 15$ and $\% 18$, respectively which shows that compared to flower pollination and krill algorithms, invasive weed algorithm has less tendency to be converged to local optimization.

\section{DISCUSSION AND CONCLUSION}

Optimization problems include a wide range of applications, especially in industrial designing, planning, timing, and etc. Real optimization problems are usually nonlinear and complicated, such that they are not solved by common mathematical methods, gradient and numerical calculations. One of the effective methods of optimization problem solving is using evolutionary algorithms inspired by the nature. In this paper, three evolutionary algorithms of invasive weed, flower pollination and krill, which are less known, were studied in terms of accuracy and convergence. Test results show that invasive weed algorithm has a better efficiency than flower pollination and krill algorithms. Moreover, Mehrabian and Lucas (2006) also showed that invasive weed algorithm has better convergence compared to particles, genetics, differential difference and frog jump algorithms.

Considering the proper convergence of invasive weed algorithm compared to other evolutionary algorithms, the future paper aims at presenting an improved version of this algorithm that can also pollinate so that it will increase in accuracy and convergence.

\section{REFERENCES}

[1] Yang, X. S. (2010). Engineering optimization: an introduction with metaheuristic applications. John Wiley \& Sons.

[2] Dasgupta, D., \& Michalewicz, Z. (Eds.). (2013). Evolutionary algorithms in engineering applications. Springer Science \& Business Media.

[3] Ghose, R., Das, T., Saha, A., Das, T., \& Chattopadhyay, S. P. (2015, October). Cuckoo search algorithm for speech recognition. In Computing and Communication (IEMCON), 2015 International Conference and Workshop on(pp. 1-5). IEEE.

[4] Tripathy, M., \& Mishra, S. (2015). Coordinated tuning of PSS and TCSC to improve Hopf Bifurcation margin in multimachine power system by a modified Bacteria Foraging Algorithm. International journal of electrical power \& energy systems, 66, 97-109.

[5] Mehrabian, A. R., \& Lucas, C. (2006). A novel numerical optimization algorithm inspired from weed colonization. Ecological informatics, 1(4), 355-366.

[6] Yang, X. S. (2012). Flower pollination algorithm for global optimization. InUnconventional computation and natural computation (pp. 240-249). Springer Berlin Heidelberg.

[7] Gandomi, A. H., \& Alavi, A. H. (2012). Krill herd: a new bio-inspired optimization algorithm. Communications in Nonlinear Science and Numerical Simulation, 17(12), 4831-4845.

[8] Gharavian, L., Yaghoobi, M., \& Keshavarzian, P. (2013, April). Combination of krill herd algorithm with chaos theory in global optimization problems. In AI \& Robotics and 5th RoboCup Iran Open International Symposium (RIOS), 2013 3rd Joint Conference of (pp. 16). IEEE

[9] ang, G. G., Gandomi, A. H., \& Alavi, A. H. (2014). Stud krill herd algorithm. Neurocomputing, 128, 363-370.

[10] Moré, J. J., \& Wild, S. M. (2009). Benchmarking derivative-free optimization algorithms. SIAM Journal on Optimization, 20(1), 172191. 